\title{
IMPLICACIÓN LABORAL DE LOS DOCENTES DE POSGRADO Y LA EVALUACIÓN QUE LES REALIZAN SUS ESTUDIANTES EN UNA UNIVERSIDAD ECUATORIANA
}

\author{
GRADUATE LECTURER'S WORK ENGAGEMENT \\ AND THE EVALUATION CARRIED OUT BY THEIR STUDENTS \\ IN AN ECUADORIAN UNIVERSITY
}

Diego Fabián Raza Carrillo?

\begin{abstract}
Resumen
El objetivo de esta investigación es identificar si existe diferencia en la evaluación que realizan los estudiantes de posgrado a sus docentes cuando reportan distintos niveles de implicación laboral en sus dimensiones de vigor, dedicación y absorción. Se tomó como referencia a los estudiantes y docentes que recibieron y dictaron clase en el tercer trimestre del año lectivo 2016-2017 en un área académica de una universidad de posgrado en Ecuador. Se utilizó las evaluaciones a docentes realizadas por los estudiantes al finalizar el trimestre, y se las contrastó con los niveles de implicación laboral reportados por sus docentes antes de iniciar el mismo trimestre. La metodología aplicada fue de enfoque cuantitativo: se aplicaron pruebas estadisticas para probar la significancia de las diferencias encontradas en los distintos análisis, y el resultado fue que los docentes que reportaron valores más altos de vigor, fueron evaluados con calificaciones más altas en su capacidad de generar un ambiente de participación en el aula. La conclusión del estudio fue que una de las dimensiones del engagement ${ }^{2}$ laboral docente tuvo incidencia en uno de los componentes de la evaluación que realizaron los estudiantes a sus docentes en la muestra seleccionada.
\end{abstract}

Palabras clave

Implicación laboral, docencia de posgrado, evaluación al docente, Ecuador.

\begin{abstract}
The goal of this research is to identify if there is a difference in the evaluation that graduate students make of their teachers when they report different levels of work engagement in their dimensions of vigor, dedication and absorption. The students and teachers who received and taught in the third quarter of the 2016-2017 school year were taken as a reference in an academic area of a graduate university in Ecuador. The evaluations of teachers carried out by the students at the end of the term were used, and they were contrasted with the levels of work engagement reported by their teachers before the beginning of the term. The methodology was quantitative in nature: statistical tests were applied to test the significance of the differences found in the different analyzes, and the result was that teachers who reported higher values of vigor were evaluated with higher scores in their ability to generate an atmosphere of participation in the classroom. The conclusion of the study is then that one of the dimensions of proffesors' work engagement had an impact on one of the components of the evaluation that students carried out on their lecturers in the selected sample.
\end{abstract}

Keywords

Work engagement, graduate professors, evaluation to professors, Ecuador

JEL: M12, M54, 123

$1 \quad$ Universidad Andina Simón Bolívar, Quito-Ecuador (diego.raza@uasb.edu.ec).

2 El work engagement se traduce usualmente como "implicación laboral", si bien en español también es usual utilizar la expresión "engagement laboral". 


\section{Introducción}

La educación superior es la que se imparte en una Universidad o institución de tercer y cuarto nivel. Es considerada como uno de los instrumentos más importantes para el desarrollo individual, social y económico de una nación; su principal propósito es la creación de conocimiento y su diseminación para el desarrollo del mundo por medio de la innovación y la creatividad (Weerasinghe et al., 2017).

Las universidades son evaluadas permanentemente en relación con su infraestructura, gestión, docencia, investigación y vinculación con la colectividad, entre otros aspectos que se consideran en los distintos modelos de evaluación aplicados, que se van perfeccionando y cambian en el tiempo, adaptándose a las distintas realidades de la educación superior y la sociedad en los distintos países del mundo y, por supuesto, también en Ecuador (Raza, 2019).

Para responder a estas evaluaciones que generalmente se realizan desde el aparato estatal, las universidades realizan con frecuencia ejercicios de autoevaluación, que a pesar de tener limitaciones de distinto tipo como, por ejemplo, de comunicación (Raza, 2020), constituyen en elementos de insumo valiosos para la mejora continua, ya que en general se acepta que dichos procesos de evaluación no solo buscan identificar la bondad de los procesos educativos, sino sobre todo sus posibilidades de mejoramiento (Cano, 1998).

Se busca entonces, el mejoramiento en el avance y progreso en el aprendizaje de los estudiantes, en la gestión universitaria y en los programas de estudio ( $R$. Lindsay et al., 2002), así como el aseguramiento en el nivel de educación impartido, entre otros propósitos que se plantean en este proceso (G. Lindsay, 2015; Love, 2010).

Uno de los mecanismos más frecuentes utilizados por las universidades para rea- lizar esta evaluación interna, en uno de sus frentes de interés, es la evaluación que realizan los estudiantes a sus docentes a través de cuestionarios o encuestas periódicas que buscan identificar, desde la perspectiva del estudiante, aquellos elementos de la labor docente que podrían mejorar.

Estas encuestas, consideran la evaluación de distintos elementos constitutivos del trabajo docente y sus resultados suelen ser considerados para decisiones de distinto tipo: permanencia o cambio de docentes, revisión de contenidos de asignaturas, atención a necesidades de formación docente en metodologías de enseñanza o sistemas de evaluación, revisión y cambio de bibliografía utilizada, trabajo en mejorar la comunicación del docente con sus estudiantes, entre muchas otras.

Entretanto, si se evalúa la gestión del docente, resulta importante preguntarse qué elementos del perfil psicosocial del docente inciden en el logro de una mayor o menor evaluación por parte de los estudiantes, entre ellos: su work engagement (que en adelante traduciremos como implicación laboral), y sus dimensiones constitutivas de vigor, dedicación, y absorption (que en adelante se traducirá como enfoque).

El artículo se organiza presentando una revisión de la literatura relacionada a la evaluación docente y a la implicación laboral, que desemboca en un planteamiento de la hipótesis de trabajo, luego de lo cual se plantea el método y análisis de datos que se aplicará, los resultados obtenidos, la discusión de dichos resultados, y algunas conclusiones para futuros estudios.

En la revisión de la literatura relacionada primero se revisan los conceptos clásicos de implicación laboral y sus elementos constitutivos; así como, una referencia a los estudios que dan cuenta de su importancia en los resultados organizacionales, y las distintas propuestas que se han realizado 
respecto de los elementos que incidan en que los trabajadores muestren mayores 0 menores niveles de implicación. De manera complementaria, en la revisión de la literatura, también se reseña la importancia de la evaluación que hace el estudiante en los procesos educativos como una forma de gestionar la calidad al interior de los centros de enseñanza.

Una vez revisada la literatura, el estudio plantea la metodología y tratamiento de los datos, dando cuenta del tipo de investigacion, enfoque, número y características de los docentes y estudiantes consultados, los instrumentos utilizados para levantar la información, y finalmente el tratamiento estadístico aplicado a la data para obtener los resultados.

Finalmente se exponen los resultados estadísticos del estudio, y se realiza una discusión de los mismos a la luz de la teoría y los antecedentes temáticos, para concluir planteando ciertas recomendaciones para la gestión en las instituciones de enseñanza.

\section{Revisión de literatura e hipótesis de trabajo}

\section{Implicación laboral}

La implicación laboral, según Schaufeli y Bakker (2004), se refiere a un estado positivo del trabajador en el que muestra un alto nivel de energía y resiliencia (vigor), un importante involucramiento en la tarea (dedicación) y una facultad para estar completamente absorto en el trabajo (enfoque). Si bien esta definición tiene más de 15 años, se la utilizará en razón de que es la que actualmente sigue siendo la de mayor acogida y la que da lugar al instrumento de medición que se sigue utilizando de manera frecuente para evaluar el nivel de implicación laboral ( Schaufeli y Bakker, 2011), que igualmente se aplica en esta investigación.
Los empleados que notoriamente están más conectados con su trabajo, absortos en él, y aplicando con energía o vigor todos sus recursos para su ejecución, en general se desempeñan mejor $y$, por tanto, generan mejores resultados organizacionales a través de un servicio mejor percibido por los beneficiarios, porque cometen menos errores $y$ muestran más cuidado en su trabajo, lo que les lleva en general a ser mejor calificados por sus supervisores (Rich et al., 2010).

De hecho, es aceptado comúnmente que las distintas dimensiones de la implicación laboral impactan en los resultados organizacionales (Bakker y Demerouti, 2007), especialmente en los relacionados con los clientes o usuarios en organizaciones de servicio (Siddiqi, 2015). Un estudio realizado en 800 unidades de negocio de 36 empresas muestra que en general los niveles de implicación laboral y sus distintas dimensiones están positivamente relacionados con el desempeño de las unidades de negocio (satisfacción del cliente, lealtad del cliente, nivel de ganancias, productividad, entre otros indicadores), y que, por tanto, se puede considerar que un funcionario con alto nivel de implicación laboral en efecto es una ventaja competitiva para la organización (Harter et al., 2002).

Parece natural aceptar que un trabajador que muestra vigor y dedicación y que tiene capacidad de estar enfocado en su trabajo, seguramente generará resultados positivos para sí mismo, para el grupo con el que trabaja y para la organización.

Desde los primeros estudios sobre implicación laboral, uno de los elementos que siempre fue de interés de los investigadores, se refirió a conocer las razones por las cuales una persona muestra mayor o menor implicación en su trabajo.

Así, el mismo Kahn en su investigación de 1990 refirió tres condiciones psicológicas determinantes para que las personas mues- 
tren mayor o menor implicación laboral: sentido (que el trabajo reporte un retorno al ser cuando se cumple el rol), seguridad (ser capaz de mostrar el propio ser sin miedo a consecuencias negativas, pérdida de imagen o estatus), y disponibilidad (sentir que se cuenta con los recursos físicos, emocionales y psicológicos para cumplir el rol).

Años antes de las propuestas de Kahn, Hackman y Oldham (1976) ya sugerían en su teoría de las características del trabajo, que las características que presenta el entorno en el que se lleva a cabo un trabajo incide en la motivación que siente la persona que lo desempeña. Esa motivación, ocasionada por algunas características del trabajo, incide en la implicación laboral, según defiende el mismo Kahn y también lo argumentan Macey y Schneider (2008).

La motivación que incide en la implicación laboral tiene que ver con la existencia de autonomía, variedad de tareas, significado de la tarea, retroalimentación, posibilidad de resolución de problemas, complejidad, entre otras, según reportan Christian, Garza y Slaughter (2011).

Estas referencias invitan a pensar que parte importante de la implicación laboral que puede presentar un trabajador, tiene que ver con el tipo de trabajo en el que se desempeñan las tareas del individuo.

Trabajos con poca variedad o muy repetitivos, trabajos con tareas que no aporten sentido de realización o significado, trabajos poco complejos en los que no se requiere creatividad para solucionar problemas, parecerían ser, por sus características, determinantes de una baja. De igual forma, trabajos en un entorno que no favorezca la autonomía, la posibilidad de intentar nuevas cosas y que no provean suficiente retroalimentación, incidirían en un implicación laboral baja de las personas que lo realizan.

En cuanto al rol del líder en la construcción de implicación laboral, Macey y
Schneider (2008) plantean que su responsabilidad es proveer un sentido de claridad en las expectativas que tiene del trabajo de su subordinado, actuar con justicia y reconocer cuando exista un buen desempeño. Aquello, según los autores, genera un sentido de vínculo con el trabajo que generaría implicación laboral en las personas.

Esto es perfectamente entendible si se considera que un jefe con esas características seguramente infundirá un sentimiento de confianza y valoración del trabajo propio en el empleado, lo cual generaría que se esfuerce un poco más en usar sus recursos físicos, emocionales, cognitivos, en el desempeño de su trabajo.

Finalmente, algunos autores otorgan un rol importante a las características personales del individuo, a la hora de mostrar implicación laboral. Hirschfeld y Thomas (2008) por ejemplo, defienden que la capacidad de la persona de regular sus propias emociones y pensamientos, permitiría a las personas lograr niveles superiores de implicación laboral. Esta propuesta tiene cercana relación con los postulados de Bandura (1992) relacionados con la auto-eficacia como capacidad de considerarse a sí mismo capaz de lidiar con cualquier dificultad.

Esta última afirmación, significaría que aun cuando el trabajo tenga características adecuadas y exista un buen líder, aquello podría no ser suficiente para generar implicación laboral en los empleados, si ellos no tienen ciertas características personales que se consideran como claves para aquello. También significaría que una persona con una alta autoeficacia, podría mostrar implicación laboral alta pese a las características de su trabajo y de su jefe, que podrían no ser las mejores.

\section{Evaluación docente}

Hill (1995) considera que la mejor manera de asegurar la calidad de la educación es gestionar el alineamiento entre las expectativas 
de sus principales "usuarios" - sus estudiantes-y su percepción del "servicio" efectivamente recibido, lo cual es complementado por Elliott y Shin (2002) que advierten sobre la cada vez mayor competencia entre instituciones de educación superior, que les obliga a preocuparse por cumplir cada vez mejor las expectativas de sus estudiantes.

Y si bien Skea (2017) alerta con toda razón sobre la posible contradicción entre lo que debe considerarse lo fundamental en los procesos educativos y el riesgo de plantear equivocadamente la relación del estudiante con la institución educativa como meramente clientelar, no se puede dejar de reconocer la importancia de comprender mejor la manera en la que la institución cumple con las expectativas del estudiante, especialmente en lo académico, para lo cual hay un acuerdo general respecto de que lo más importante en este proceso es la evaluación que se hace a los aspectos académicos (relación con el docente, perfil del docente, experiencia de aprendizaje en general), más que a aquellos logísticos o administrativos (Thomas y Galambos, 2004).

A las universidades les resulta importante asegurar que la experiencia estudiantil sea adecuada en su proceso de formación, considerando que para el estudiante esta experiencia tiene varios componentes y su carácter es multidimensional y multicausal, incidiendo en ella la calidad de la educación que reciben, la idoneidad del acompañamiento que le ofrece el equipo académico, la malla curricular y contenidos de cada materia, la metodología con la que reciben su formación, los servicios de apoyo y soporte, la infraestructura física y otros (Wiers-Jenssen et al., 2002).

La experiencia general que viven los estudiantes es además modulada por su estado emocional (posible estrés, depresión, ansiedad, frustración), y la forma en la que perciben el soporte social que reciben de la universidad (Pidgeon et al., 2017).

Pese a que varios estudios muestran que la simple medición o consideración de la calidad a partir de la satisfacción del estudiante, no necesariamente conlleva a mejores niveles de aprendizaje (Cheng et al., 2016), a lo largo de los años se han planteado distintos modelos de medición de esta experiencia en la educación superior, con propuestas que han evolucionado de manera constante desde la década de 1970 hasta nuestros días (Weerasinghe et al., 2017), y en las que uno de los factores comunes, entre todas esas propuestas, es el interés en evaluar al docente, por su alta incidencia tanto en la experiencia estudiantil (Anagnostopoulou et al., 2015), cuanto en el juicio que realiza el estudiante respecto del cumplimiento de sus expectativas de la clase, que determinará su satisfacción con el proceso (Mikulić et al., 2015).

Uno de los resultados organizacionales que se buscan usualmente en las universidades es lograr una buena evaluación a sus docentes por parte de sus estudiantes; en este estudio se busca identificar si esa evaluación es diferente cuando los docentes presentan distintos niveles de implicación laboral.

\section{Método y análisis de datos}

El paradigma investigativo en el que se enmarca esta investigación es principalmente el del pospositivismo, en tanto a través de un enfoque cuantitativo se interesa en determinar posibles causas a los fenómenos de estudio (Creswell, 2014), por medio de un estudio de tipo explicativo que trata de aportar a la comprensión de la relación y posible causalidad de variables (Bernal, 2010), y que de manera particular indaga sobre la posible incidencia de la implicación laboral de los docentes en la evaluación que hacen los estudiantes de la labor de esos docentes en un 
entorno de posgrado, con un enfoque hipotético-deductivo que sugiere la posibilidad de ir abonando en la generalización de resultados particulares (Phillips y Pugh, 2008).

Para ello se llevó a cabo un estudio de tipo ex post facto que consiste en la comparación de dos o más grupos que no han sido intencionalmente intervenidos por el investigador (Ravid, 2011) en el que se compararon los niveles de evaluación realizados por los estudiantes de sus docentes, cuando aquellos presentan niveles más altos o más bajos de implicación laboral en sus distintas dimensiones.

Este estudio se llevó a cabo en una universidad de posgrado en la ciudad de Quito en Ecuador, específicamente en una de sus áreas académicas, la de mayor número de estudiantes.

Se solicitó información de los 31 docentes que dictaron clase en el tercer trimestre del año lectivo 2016-2017 en esa área, a través de una nota escrita por parte del director del área, informando el propósito del estudio y solicitando su participación. Por distintas razones, cinco de ellos se excusaron de participar en el estudio, por lo que finalmente se trabajó con la información de 26 de los 31 docentes del área en el trimestre.

De manera concordante, se consideró a los 861 estudiantes que recibieron clase con los 26 profesores, de los cuales 784 $(91,06 \%)$ cumplieron con llenar el reporte de evaluación al docente que fue aplicado por la universidad al terminar el tercer trimestre del año lectivo 2016-2017.

Para recoger información del nivel de implicación laboral, el cuestionario se aplicó de manera personal a cada uno de los 26 docentes por parte del investigador en las instalaciones de la propia universidad, para recoger los resultados de la evaluación al docente que realizaron los estudiantes, se solicitó a la Dirección General Académica de la Universidad que proporcione los datos que regularmente recogen con este fin, específicamente los correspondientes al trimestre académico en el que se realizó el estudio.

Para medir la implicación laboral se utilizó la escala UWES (W. Schaufeli y Bakker, 2011), que contiene tres dimensiones que son vigor, dedicación y absorción o enfoque, y que se miden a través de 17 preguntas. Las respuestas se consignaron en una escala de Likert de 6 puntos en los que 0 es nunca, 1 es casi nunca, 2 es algunas veces, 3 es regularmente, 4 es bastantes veces, 5 es casi siempre, y 6 es siempre.

Para medir la evaluación que realizan los estudiantes al docente se utilizó la escala de evaluación propia de la universidad que contiene seis preguntas, de las cuales cuatro tienen relación directa con la labor del docente en el aula, ${ }^{3} y$ que se refieren a su capacidad de entrelazar los conceptos en la asignatura que tiene a cargo, la profundidad de conocimiento que demuestra tener sobre dichos conceptos, su habilidad de generar un ambiente participativo en el aula, y la transparencia de sus criterios de evaluación. Las respuestas se consignaron en una escala de Likert de 5 puntos en los que 1 es calificación mínima y 5 es calificación máxima.

Los datos recogidos fueron analizados utilizando el programa estadístico SPSS. Se aplicaron pruebas de Shapiro Wilk a las dos variables (implicación laboral y evaluación al docente) y a sus dimensiones constitutivas, con el fin de determinar su normalidad, y en función del resultado de esta prueba, se debió aplicar pruebas $U$ de Mann Whitney con el fin de determinar si las diferencias encontradas en las medianas de las distintas variables eran estadísticamente significativas.

Para complementar y contextualizar los resultados estadísticos se conformó un

3 Las otras dos preguntas evalúan elementos de apoyo administrativo académico, por tanto, se las excluye por no evaluar la labor del docente en el aula. 
grupo focal con estudiantes de los distintos programas del área académica en la que se realizó el estudio para indagar respecto de los determinantes de una mayor o menor evaluación a sus docentes.

\section{Resultados}

La tabla 1 a continuación muestra los valores mínimos y máximos, así como las medianas de cada una de las dos variables de estudio y sus respectivos componentes.

Tabla 1. Mínimo, máximo y mediana de las variables del estudio

\begin{tabular}{|l|c|c|c|}
\hline & Mínimo & Máximo & Mediana \\
\hline Vigor & 17 & 36 & 31 \\
\hline Dedicación & 12 & 30 & 27,5 \\
\hline Absorción o enfoque & 11 & 36 & 30 \\
\hline Implicación laboral & 47 & 102 & 88,5 \\
\hline Nexos & 3,4 & 4,83 & 4,55 \\
\hline Dominio & 3,76 & 4,97 & 4,64 \\
\hline Participación & 3,16 & 4,83 & 4,465 \\
\hline Claridad & 3,4 & 4,93 & 4,5 \\
\hline ETD $^{4}$ & 13,72 & 19,26 & 18,205 \\
\hline
\end{tabular}

Elaboración: el autor.

Los valores mínimos, máximos y de medianas del "vigor", "dedicación", "absorción o enfoque" e "implicación laboral" son los correspondientes a los reportados por los 26 docentes a través del instrumento UWES cuyas preguntas se respondieron, como ya se ha dicho, con una escala de Likert de 6 puntos.

Los valores mínimos, máximos y de medianas de "nexos", "dominio", "participación", "claridad" y "ETD" son los correspondientes a las calificaciones (en una escala de 5 puntos) que consignaron los 784 estudiantes que completaron la evaluación para los mismos docentes.
Se aplicó la prueba Shapiro Wilk para determinar la normalidad de los datos recogidos de implicación laboral, vigor, dedicación y absorción o enfoque del docente, con el fin de contar con elementos de decisión para el uso de pruebas paramétricas o no-paramétricas en los análisis posteriores de diferencia.

El resultado de la aplicación de la prueba Shapiro Wilk para determinar la normalidad de los datos recogidos tanto de implicación laboral (y cada una de sus dimensiones) como de la evaluación docente que hacen los estudiantes (en cada una de sus dimensiones), aparecen a continuación en la tabla 2 y tabla 3.

Tabla 2. Prueba Shapiro Wilk para determinar normalidad en implicación laboral y sus dimensiones

\begin{tabular}{|l|l|l|l|}
\hline \multicolumn{1}{|c|}{ Variable } & \multicolumn{1}{c|}{ Estadístico } & \multicolumn{1}{c|}{ Valor de $\mathrm{p}$} & \multicolumn{1}{c|}{ Resultado } \\
\hline Implicación laboral & $\mathrm{z}=3,241$ & $\mathrm{p}<0,01$ & No es normal \\
\hline Vigor & $\mathrm{z}=2,69$ & $\mathrm{p}=0,004$ & No es normal \\
\hline Dedicación & $\mathrm{z}=4,15$ & $\mathrm{p}<0,001$ & No es normal \\
\hline Absorción o enfoque & $\mathrm{z}=2,779$ & $\mathrm{p}=0,003$ & No es normal \\
\hline
\end{tabular}

Elaboración: el autor.

$4 \quad$ Evaluación total que se hace al docente. 
Tabla 3. Prueba Shapiro Wilk para determinar normalidad en la evaluación que hace el estudiante al docente y sus componentes

\begin{tabular}{|l|l|l|l|}
\hline \multicolumn{1}{|c|}{ Variable } & \multicolumn{1}{c|}{ Estadístico } & \multicolumn{1}{c|}{ Valor de $p$} & \multicolumn{1}{c|}{ Resultado } \\
\hline ETD & $\mathrm{z}=3,50$ & $\mathrm{p}<0,001$ & No es normal \\
\hline Nexos & $\mathrm{z}=3,76$ & $\mathrm{p}<0,001$ & No es normal \\
\hline Dominio & $\mathrm{z}=3,64$ & $\mathrm{p}<0,001$ & No es normal \\
\hline Participación & $\mathrm{z}=2,68$ & $\mathrm{p}=0,004$ & No es normal \\
\hline Claridad & $\mathrm{z}=3,45$ & $\mathrm{p}<0,001$ & No es normal \\
\hline
\end{tabular}

Elaboración: el autor.

Nota: $\mathrm{ETD}$ = Evaluación total que se hace al docente.

Como se puede concluir de los datos presentados en las dos tablas anteriores, ninguna de las dos variables que son objeto de esta investigación, la implicación laboral y la evaluación que hace el estudiante al docente, ni ninguno de sus elementos constitutivos, muestran un comportamiento de distribución normal, y por tanto, para el análisis de diferencias de sus medidas de tendencia central, se deben aplicar pruebas no paramétricas.

\section{Evaluación que realiza el estudiante al docente, y sus componentes, con distinto nivel de implicación laboral}

Para evaluar la diferencia en la evaluación que hace el estudiante al docente cuando reporta niveles más altos en las distintas dimensiones de la implicación laboral, se compararon los valores reportados de im- plicación laboral, vigor, dedicación y absorción, en los niveles reportados de evaluación estudiantil al docente (y sus componentes) más altos y más bajos.

Es decir, se dividieron en dos grupos las observaciones en cada componente, separando las evaluaciones más altas de las más bajas, y en cada mitad, se evaluó cuál fue el valor (la mediana) de implicación laboral, vigor, dedicación y absorción o enfoque reportado por los docentes. Se compararon los dos grupos y se determinó si la diferencia en cada dimensión de la implicación laboral entre los profesores mejor evaluados por sus estudiantes y los de evaluación más baja, fue significativa estadísticamente.

Para determinar la significancia estadística se aplicó una prueba de Mann Whitney a cada diferencia evaluada, sus resultados aparecen en la tabla a continuación.

Tabla 4. Resultados de la prueba de Mann-Whitney aplicada para evaluar diferencias de implicación laboral en niveles altos y bajos de evaluación que hacen los estudiantes a sus docentes, y sus componentes

\begin{tabular}{|c|c|c|c|c|c|c|c|c|}
\hline & Implicaci & & & & & & & \\
\hline & Valor prueba & $\mathrm{p}$ & leba & $p$ & Valor prueba & $\mathrm{p}$ & Valor prueba & $\mathrm{p}$ \\
\hline al & $13,13)=54,0$ & 25 & 51,5 & 091 & $=53,5$ & 113 & $=69,0$ & 0,448 \\
\hline conte & $=58,5$ & 217 & $=53,5$ & 134 & $\mathrm{U}(11$ & 0,198 & $=70,5$ & 0,54 \\
\hline omir & $\mathrm{U}(12$, & 0,560 & $U(12,14)=69,0$ & 0,462 & $U(12,14)=70,5$ & 0,494 & $\mathrm{U}(12,1$ & 0,899 \\
\hline perti & 13 & 0,960 & $U(13,13)=79,5$ & 0,801 & $U(13,13)=66,5$ & 0,362 & $U(13,1$ & 0,801 \\
\hline ene & $\mathrm{U}(13$, & 0,072 & $=42,5$ & 0,029 & $U(13,13)=54,5$ & 0,125 & $\mathrm{U}(13$, & 0,336 \\
\hline Claridad criterios evalu & $U(12,14)=77,0$ & 0,742 & $U(12,14)=74,5$ & 0,631 & $U(12,14)=80,5$ & 0,860 & $U(12,14)=81$ & 0,899 \\
\hline Cumplimiento programa & $U(12,14)=84,0$ & 1,000 & $U(12,14)=83,0$ & 0,980 & $U(12,14)=80,5$ & 0,860 & $U(12,14)=75$ & 0,667 \\
\hline
\end{tabular}

Elaboración: el autor. 
En todos los casos la Ho fue que no existía diferencia en la evaluación que realizan los estudiantes a los docentes (en sus distintos componentes), atribuible al distinto nivel (mayor o menor) de implicación laboral y sus elementos constitutivos reportados por los docentes. De manera concordante la Ha fue que sí existe diferencia en estas evaluaciones. Se tomó el valor de $p<=0,05$ como valor de referencia para rechazar la hipótesis nula.

Los resultados de la prueba muestran que las únicas diferencias estadísticamente significativas se dan en el cruce de las variables "capacidad de generar participación" y "vigor". Esto significa que la evaluación que realizan los estudiantes al docente, relativa a su capacidad de generar un clima de participación en el aula, fue mayor estadísticamente cuando los valores reportados de vigor por parte de los docentes eran más altas, como se muestra en la gráfica a continuación.

Ilustración 1. Evaluación de la capacidad de generar participación por parte de docentes que reportan distintos niveles de vigor

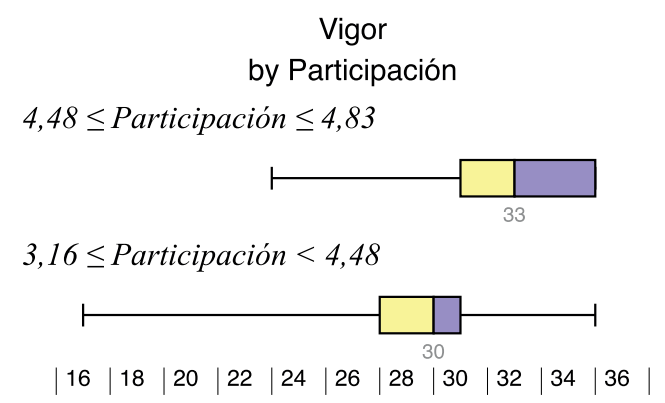

Elaboración: el autor.

El gráfico indica los resultados obtenidos al procesar la información recolectada y luego de haber aplicado las pruebas estadísticas: los profesores cuya capacidad de generar un clima de participación en el aula fue evaluada con valores más altos (de 4,48 a 4,83 sobre 5 versus 3,16 a menos de 4,48 sobre 5), reportaron un nivel de vigor esta- dísticamente más alto que el resto de docentes (medianas de 33 versus 30).

Los resultados de la prueba en la evaluación de diferencias de las distintas variables (tabla 4), muestran además que los distintos niveles de dedicación, absorción o enfoque e implicación reportados por los docentes, no son diferentes estadísticamente en los distintos niveles de evaluación docente que realizan los estudiantes.

\section{Discusión de los resultados}

Resulta interesante que el único par de variables analizado que obtuvo resultados significativos estadísticamente fue el de vigorparticipación con el análisis realizado. Esto significa que de todos los elementos constitutivos de la implicación laboral (vigor, dedicación, absorción o enfoque), solo los distintos niveles de vigor en el docente muestran una evaluación mayor del estudiante hacia el docente; y además solo en uno de los elementos evaluados, que es la evaluación que hicieron los estudiantes a la capacidad del docente de generar un clima de participación en el aula.

Resulta interesante que, en el grupo focal realizado con estudiantes, justamente fuera esta relación la única que se mencionó espontáneamente por parte de los estudiantes, que consideraron no solo que de los elementos de evaluación mencionados uno de los más importantes es el de la capacidad de generar participación, sino que reconocían que esta participación tenía relación directa con el nivel de energía física y emocional que percibían en el docente durante las clases.

Esta "energía" -que se refiere directamente al vigor - manifestaron los estudiantes que se revela justamente en poder involucrar a los estudiantes en las discusiones, conceptualizaciones, ejercicios de clase -grupales e individuales-, y hacer de esta participación un elemento metodológico en el proceso enseñanza-aprendizaje. 
Los estudiantes también opinaron que los otros elementos de su evaluación (claridad en los criterios de evaluación, cumplimiento del programa, conocimiento de la materia, pertinencia de la bibliografía, capacidad de establecer nexos entre los contenidos), no advertían qué relación podrían tener con los niveles de "energía" del docente, lo cual guarda coherencia con los resultados estadísticos de este estudio.

En el mismo grupo focal, los estudiantes restaron importancia a los otros elementos constitutivos de la implicación laboral de sus docentes en relación con la evaluación que hacen sus estudiantes respecto de su labor. La dedicación (referida a la vocación, inspiración, orgullo de dictar clase), y la absorción o enfoque (capacidad de abstraerse en la tarea por parte del docente) no pudo ser identificada por los estudiantes como elementos que determinen una percepción de mejor nivel en los elementos constitutivos de su evaluación realizada a los docentes, por cuanto consideraron que es muy similar en todos los docentes, y que su nivel no determina necesariamente que el profesor haga participar más, o genere mejores nexos en los contenidos, o que tenga alguna relación con la pertinencia del syllabus.

Los resultados estadísticos, y la contrastación hecha por medio del grupo focal con estudiantes, parece que permitiría pensar que lo que genera una mejor evaluación del estudiante hacia el docente, es el vigor con el que el profesor se esfuerza en generar momentos participativos en el aula.

\section{Conclusiones y líneas futuras de investigación}

Si bien existe literatura abundante que relaciona el engagement o implicación laboral con los resultados organizacionales (Bakker y Demerouti, 2007; Harter et al., 2002; Rich et al., 2010; Siddiqi, 2015), este estudio abo- na con elementos que permiten pensar que esta relación al parecer también se cumple en instituciones de educación superior, específicamente se ha verificado que la evaluación que hacen los estudiantes, de la capacidad del docente para generar un clima participativo en el aula, es más alta cuando el docente reporta tener mayor vigor, como uno de los elementos constituyentes de su engagement o implicación laboral.

No se debe perder de vista que este estudio ha tenido varias limitaciones: la muestra fue limitada a una sola de las áreas académicas de la Universidad estudiada, y solo a lo ocurrido con estudiantes y docentes en un trimestre académico específico en un año lectivo en particular, y asimismo el análisis se ha limitado a observar el posible impacto del engagement o implicación laboral del docente en la evaluación que hace el estudiante del docente.

Estas limitaciones invitan a realizar a futuro investigaciones complementarias que busquen verificar los resultados en otras áreas académicas o facultades, en otras universidades y países, y que indaguen sobre otro tipo de relaciones en búsqueda de mayor potencia estadística que ratifique -o cuestione- estos resultados. De igual forma, parece necesario llevar a cabo otras investigaciones que busquen indagar respecto de la incidencia de la implicación laboral docente en otras variables relacionadas con el proceso de enseñanza aprendizaje en la educación de tercer y cuarto nivel.

\section{Referencias}

Anagnostopoulou, E.; Mavroidis, I.; Giossos, Y. y Koutsouba, M. (2015). Student satisfaction in the context of a postgraduate programme of the Hellenic Open University. Turkish Online Journal of Distance Education, 16(2), 40-55. https://doi.org/10.17718/tojde.52944 
Bakker, A. B. y Demerouti, E. (2007). The Job Demands-Resources model: State of the art. Journal of Managerial Psychology, 22(3), 309-328. https://doi.org/10.11 08/02683940710733115

Bandura, A. (1992). Exercise of personal agency through the self-efficacy mechanism. En Self-efficacy: Thought control of action (pp. 3-38). Hemisphere Publishing Corp.

Bernal, C. (2010). Metodología de la investigación: Administración, economía, humanidades y ciencias sociales (3. ${ }^{a}$ ed.). Pearson Educación.

Cano, E. (1998). Evaluación de la calidad educativa. Editorial La Muralla.

Cheng, M.; Taylor, J.; Williams, J. y Tong, K. (2016). Student satisfaction and perceptions of quality: Testing the linkages for PhD students. Higher Education Research \& Development, 35(6), 1153-1166. https://doi.org /10.1080/07294360.2016.1160873

Christian, M. S.; Garza, A. S. y Slaughter, J. E. (2011). Work engagement: A quantitative review and test of its relations with task and contextual performance. Personnel Psychology, 64(1), 89-136. https://doi. org/10.1111/j.1744-6570.2010.01203.x

Creswell, J. W. (2014). Research Design: Qualitative, Quantitative, and Mixed Methods Approaches. SAGE.

Elliott, K. M. y Shin, D. (2002). Student Satisfaction: An alternative approach to assessing this important concept. Journal of Higher Education Policy and Management, 24(2), 197-209. https://doi.org/10.1080/ 1360080022000013518

Hackman, R. y Oldham, G. (1976). Motivation through the Design of Work: Test of $A$ Theory. Organizational Behavior and Human Decision Processes, 16.

Harter, J. K., Schmidt, F. L. y Hayes, T. L. (2002). Business-Unit-Level Relationship Between Employee Satisfaction, Employee Engagement, and Business Outcomes: A MetaAnalysis. Journal of Applied Psychology, 87(2), 268-279.

Hill, F. M. (1995). Managing service quality in higher education: The role of the student as primary consumer. Quality Assuran- ce in Education, 3(3), 10-21. https://doi. org/10.1108/09684889510093497

Hirschfeld, R. y Thomas, C. (2008). Representations of Trait Engagement: Integration, Additions, and Mechanisms. Industrial and Organizational Psychology, 1(1), 63-66.

Lindsay, G. (2015). Educational Evaluation: Overview. En J. D. Wright (ed.), International Encyclopedia of the Social \& Behavioral Sciences (Second Edition) (pp. 229-231). Elsevier. https://doi.org/10.1016/B978-008-097086-8.92062-2

Lindsay, R.; Breen, R. y Jenkins, A. (2002). Academic Research and Teaching Quality: The views of undergraduate and postgraduate students. Studies in Higher Education, 27(3), 309-327. https://doi.org/10.1080/ 03075070220000699

Love, A. J. (2010). Understanding Approaches to Evaluation. En P. Peterson, E. Baker, y B. McGaw (Eds.), International Encyclopedia of Education (Third Edition) (pp. 798-807). Elsevier. https://doi.org/10.1016/B978-008-044894-7.01594-3

Macey, W. y Schneider, B. (2008). The Meaning of Employee Engagement. Industrial and Organizational Psychology, 1(1), 3-30.

Mikulić, J.; Dužević, I. y Baković, T. (2015). Exploring drivers of student satisfaction and dissatisfaction: An assessment of impactasymmetry and impact-range. Total Quality Management \& Business Excellence, 26(11/12), 1213-1225. https://doi.org/10.1 080/14783363.2014.925291

Phillips, E. y Pugh, D. (2008). La tesis doctoral. Un manual para estudiantes y sus directores. Amat Editorial.

Pidgeon, A. M., Davies, N. L., y Stapleton, P. (2017). Factors Influencing University Students' Academic Experience: An International Study. International Journal of Multidisciplinary Perspectives in Higher Education, 2(1), 1-8.

Ravid, R. (2011). Practical statistics for educators (4. ${ }^{\text {a }}$ ed.). Rowman \& Littlefield.

Raza, D. (2019). Evaluación y acreditación universitaria en Ecuador. Revista de Educación Superior en América Latina, O(6). http:// dx.doi.org/10.14482/esal.6.378.766 
Raza, D. (2020). Mejorando la comunicación en los procesos de autoevaluación de la calidad en universidades. Revista Chakiñan de Ciencias Sociales y Humanidades, 10, 144-156. https://doi.org/10.37135/chk.002.10.10

Rich, B. L.; Lepine, J. A. y Crawford, E. R. (2010). Job Engagement: Antecedents and Effects on Job Performance. Academy of Management Journal, 53(3), 617-635. https://doi. org/10.5465/AMJ.2010.51468988

Schaufeli, W. B. y Bakker, A. B. (2004). Job demands, job resources, and their relationship with burnout and engagement: A multi-sample study. Journal of Organizational Behavior, 25(3), 293-315. https:// doi.org/10.1002/job.248

Schaufeli, W. y Bakker, A. (2011). Escala Utrecht de Engagement en el Trabajo $(\mathrm{H}$. Valdez \& C. Ron, Trads.). Occupational Health Psychology Unit, Utrecht University.

Siddiqi, M. A. (2015). WorkEngagement and JobCrafting of Service Employees Influencing Customer Outcomes. Vikalpa: The Journal for
Decision Makers, 40(3), 277-292. https:// doi.org/10.1177/0256090915598584

Skea, C. (2017). Student Satisfaction in Higher Education: Settling up and Settling Down. Ethics and Education, 12(3), 364-377. https://doi. org/10.1080/17449642.2017.1343560

Thomas, E. y Galambos, N. (2004). What Satisfies Students? Mining Student-Opinion Data with Regression and Decision Tree Analysis. Research in Higher Education, 45, 251-269. https://doi.org/10.1023/B:RIHE. 0000019589.79439.6e

Weerasinghe, S.; Lalitha, S. y Fernando, R. Ls. (2017). Students' Satisfaction in Higher Education Literature Review. https://doi. org/10.12691/education-5-5-9

Wiers-Jenssen, J., Stensaker, B., \& Grogaard, J. B. (2002). Student Satisfaction: Towards an empirical deconstruction of the concept. Quality in Higher Education, 8(2), 183-195. https://doi.org/10.1080/135383 2022000004377 